\title{
Photoperiod regulates the differential expression of KiSS-1 and GPR54 in various tissues and sexes of striped hamster
}

\author{
S.N. Li, H.L. Xue, Q. Zhang, J.H. Xu, S. Wang, L. Chen and L.X. Xu \\ College of Life Sciences, Qufu Normal University, Qufu, Shandong, Qufu City, \\ Shandong Province, China \\ Corresponding author: L.X. Xu \\ E-mail: xulx@mail.qfnu.edu.cn
}

Genet. Mol. Res. 14 (4): 13894-13905 (2015)

Received May 12, 2015

Accepted July 31, 2015

Published October 29, 2015

DOI http://dx.doi.org/10.4238/2015.October.29.10

ABSTRACT. In order to investigate how photoperiod affects the expression of KiSS-1/G-protein-coupled receptor 54 (GPR54), the hypothalamus, the ovaries and testicles of the striped hamster were studied following photoperiod treatment and melatonin injection. We found that the melatonin concentration in the blood of the striped hamster was consistent with the treated photoperiods and that the photoperiod had significant effects on KiSS-1 expression, but the effect of photoperiod on GPR54 expression differed among tissues and sexes. The relative expression of KiSS-1 in the gonads of the male and female striped hamster was significantly higher than that in the hypothalamus, while the difference in GPR54 between the gonads and hypothalamus was inconsistent between the male and female striped hamster. In the striped hamster injected with melatonin, the expression level of KiSS-1 in the gonads and hypothalamus of the male and female striped hamster was significantly decreased; the expression level of $\mathrm{GnRH}$ in the gonads was also significantly decreased; the expression of GPR54 in the hypothalamus was increased. In the hypothalamus, a significant positive correlation existed between KiSS-1 and $\mathrm{GnRH}$ expression, while a negative correlation was observed between 
GPR54 and GnRH expression. These results suggest that the photoperiodmelatonin-KiSS-1-GnRH pathway plays an important role in the seasonal reproduction mechanism of the striped hamster. Additionally, photoperiod or melatonin may not be a direct regulatory factor of GPR54 expression.

Key words: G-protein-coupled receptor 54 gene; KiSS-1; Melatonin; Gonadotropin-releasing hormone gene; Photoperiod

\section{INTRODUCTION}

Most wild animals restrict their fertility to a particular time of the year to ensure the birth of the offspring during the most favorable season (Simonneaux et al., 2009). The phenomenon of breeding confined to a particular season is referred to as seasonal reproduction. Seasonal reproduction is a complex biological phenomenon, which relies on both internal and external environments. Photoperiod is a typical seasonal variation that regulates reproduction in seasonal breeders, and most seasonal breeders modulate reproductive activity by responding to the photoperiod (Pévet, 1988; Bartke and Steger, 1992; Goldman, 2001; Prendergast, 2005). Photoperiodic information can be transmitted to the reproductive system by melatonin (Simonneaux et al., 2009), which is a pineal hormone that is secreted at levels which are proportional to the night length. Therefore, variation in the concentration of melatonin in animals throughout the year is an ideal and reproducible indicator of photoperiod (Simonneaux and Ribelayga, 2003). Melatonin can regulate the secretion of gonadotropin-releasing hormone $(\mathrm{GnRH})$ and luteinizing hormone $(\mathrm{LH})$ (Malpaux et al., 2002), causing seasonal changes in reproductive activity. These previous studies showed that variation in melatonin caused by photoperiod can regulate reproductive activity by modulating the secretion of $\mathrm{GnRH}$, which is the major driving signal to induce self-priming at the pituitary and to stimulate the pulsatile release of both gonadotropins, $\mathrm{LH}$ and follicle-stimulating hormone (FSH) (Roa and Tena-Sempere, 2007).

Some studies suggested that kisspeptin neurons are a part of the environmental regulatory mechanism of reproduction, which includes photoperiod regulation of the reproductive system (Parhar et al., 2012). Kisspeptin neurons have been proposed as a mediator of photoperiodic signals in the reproductive system; the effect of photoperiods is mostly mediated by melatonin (Kitahashi and Parhar, 2013). Kisspeptin was also shown to be closely correlated with the expression of GnRH (Parhar et al., 2004) and KiSS-1 in GnRH neurons in Oreochromis niloticus (Irwig et al., 2004; Messager et al., 2005). Some studies also found that kisspeptin neuronal fibers were appositional with $\mathrm{GnRH}$ cell bodies in the brain of mammals and fishes (Servili et al., 2011). These studies revealed that kisspeptin stimulates the release of $\mathrm{GnRH}$ and controls the hypothalamus-pituitary-gonadal axis in most vertebrates (Kitahashi and Parhar, 2013). Sexually inactive Syrian hamsters administered kisspeptin under photoinhibitory short day (SD) conditions restored testicular activity in the hamsters (Revel et al., 2006b), indicating that kisspeptin plays a key role in regulating the reproductive axis, and kisspeptin administration in photoinhibited animals could reactivate reproductive activity (Simonneaux et al., 2009). Based on these results, we hypothesized that the photoperiod-melatonin- kisspeptin-GnRH system exists in animals and regulates their seasonal reproduction.

Kisspeptin is the product of the KiSS-1 gene, and the interaction between kisspeptin and its receptor GPR54 plays a crucial role in regulating adult reproduction. Kisspeptin is thought to be a very potent stimulator of the $\mathrm{GnRH} /$ gonadotropin axis (Roa and Tena-Sempere, 2007). A 
previous study suggested that GPR54 also plays a role in reproduction in non-mammalian species (Roa and Tena-Sempere, 2007).

The KiSS-1/GPR54 system was found to be an important regulator of reproduction. Mutations in the GPR54 gene in humans and mice caused striking impairments in reproductive function (de Roux et al., 2003; Funes et al., 2003) and a severe deficiency in gonadotropin (LH and $\mathrm{FSH}$ ) secretion, ultimately diminishing the secretion of $\mathrm{GnRH}$. In sheep, kisspeptin infusions dramatically increased the concentration of $\mathrm{GnRH}$ in hypothalamo-pituitary portal circulation. Exogenous kisspeptin treatment may promote the secretion of LH and FSH (Gottsch et al., 2004; Irwig et al., 2004; Matsui et al., 2004; Dhillo et al., 2005; Navarro et al., 2005; Shahab et al., 2005; Greives et al., 2007; Roa and Tena-Sempere, 2007). These studies demonstrate that kisspeptinmediated mechanisms for seasonal reproduction may exist in all photoperiodic breeders (Kauffman et al., 2007).

KiSS-1 is expressed in a wide range of tissues, including the brain and ovaries. Its spatial expression in the brain varies among different species (Clarkson and Herbison, 2006; Franceschini et al., 2006; Kanda et al., 2008; Kitahashi et al., 2009). For example, KiSS-1 is expressed in the arcuate nucleus (Arc) and the anteroventral periventricular nucleus (AVPV) in the rodent brain (Clarkson and Herbison, 2006), and in the Arc and the preoptic area in the sheep brain (Franceschini et al., 2006).

The photoperiod also has differential effects on KiSS-1 expression in the AVPV among different breeds (Kitahashi and Parhar, 2013). For example, SD conditions suppress the expression of KiSS-1 in the AVPV in Syrian hamsters (Revel et al., 2006a; Ansel et al., 2010) but increase expression in the Arc of Siberian hamsters (Mason et al., 2007; Simonneaux et al., 2009). SD conditions suppress the expression of KiSS-1 in the Arc of Syrian hamsters and Siberian hamsters (Mason et al., 2007; Simonneaux et al., 2009). The effect of photoperiod on KiSS-1 expression in the ovaries is less well understood.

The striped hamster, Cricetulus barabensis, is a dominant rodent pest in northern China farmland with a high reproductive capacity. In spring (March to April) and autumn (August to September), the striped hamster shows peak reproductive activities, while no reproductive activity occurs during the winter (December to January). This species can breed 3 times every year, giving birth to 4-9 fetuses for every parity (Li et al., 1990). How the photoperiod affects the expression of KiSS-1/GPR54 in the hypothalamus, ovaries, or testicles of the striped hamster is unknown. In this study, the effect of the photoperiod on the expression of the KiSS-1/GPR54 in the hypothalamus, ovaries, and testicles in the striped hamster was studied following photoperiod treatment and melatonin injection.

\section{MATERIAL AND METHODS}

\section{Sample collection}

The striped hamsters used in this study were captured by the iron cage live-trapped method in the fields of the town of PuWang, Shandong Province, which is a long-term research base established by our research team for agricultural rodents. Trapped individuals were appraised, numbered, and fed in the animal feeding room at the experimental center for Qufu Normal University. Composite rat food particles, purchased from Jining Medical College (Jining, China), were used to feed the striped hamsters; hamsters had access to drinking water. In the feeding room, the natural light irradiate and the temperature was maintained at $22^{\circ}-25^{\circ} \mathrm{C}$. Ethical approval was granted by The Qufu Normal University's Animal Ethics Committee. 


\section{Photoperiod treatment}

Ninety female and 54 male adult striped hamsters were randomly selected; the female and male striped hamsters all had similar body weights (weight deviation did not exceed $5 \%$ ) between individuals of the same sex, and all female striped hamsters had stable estrous cycles. The hamsters were randomly divided into 3 groups, including long day (LD), control (CD), and short day (SD) groups. The LD, CD, and SD individuals were treated with photoperiods of 16L:8D, 12L:12D, and 8L:16D, respectively, for 5 weeks.

\section{Melatonin injections}

Thirty-six adult striped hamsters of each sex were randomly selected. Within the same sex, individuals showed similar body weights, with weight deviation not exceeding $5 \%$. All female individuals had stable estrous cycles. Female and male individuals were randomly divided into 2 groups, including the injection group and the control group. Injection measures were conducted daily at 19:00 for 2 weeks. Individuals from the injection group were injected with $400 \mu \mathrm{L}$ melatonin solutions (Takara Biotechnology, Dalian, China) at a concentration of $50 \mathrm{mg} / \mathrm{kg}$, and those from the control group were injected with $400 \mu \mathrm{L}$ normal saline containing $2 \%$ ethanol. During the treatment period, the photoperiod was $12 \mathrm{~L}: 12 \mathrm{D}$, the temperature was maintained at $22^{\circ}-25^{\circ} \mathrm{C}$, and drinking water was freely accessible.

\section{Tissues separation}

After treatment, striped hamsters were anesthetized using ether and sacrificed by cervical dislocation in the dark. The hypothalamus, ovary, and testis were quickly removed and frozen in liquid nitrogen, and then stored at $-80^{\circ} \mathrm{C}$ until use.

\section{Hormone determination}

Blood samples were collected immediately after sacrifice and stored at $4^{\circ} \mathrm{C}$ for 30 min, then centrifuged with $1200-2000 \mathrm{~g} / \mathrm{min}$ for $15 \mathrm{~min}$ at $4^{\circ} \mathrm{C}$. Serum melatonin levels were estimated using an enzyme-linked immunosorbent assay Labsystems Multiskan MS 352.

\section{Quantitative analysis of gene expression}

Total RNA was extracted from the hypothalamic, ovarian, and testicular tissues of the collected striped hamsters using RNA extraction kit (Takara Biotechnology, Dalian, China). The cDNA was reverse transcribed from the mRNA included in the total RNA. The amplification reaction contained the following: $1 \mu \mathrm{g}$ RNA, $2 \mu \mathrm{L} 10 \mathrm{mM}$ dNTPs, and $1 \mu \mathrm{L}(50 \mathrm{pM})$ Oligo $\mathrm{dT}_{18}$. Denaturation was carried out at $70^{\circ} \mathrm{C}$ for $10 \mathrm{~min}$ and then quenched on ice. Next, $4 \mu \mathrm{L} 5 \mathrm{X}$ reverse transcriptase buffer, $1 \mu \mathrm{L}$ AMV reverse transcriptase, $0.5 \mu \mathrm{L}$ RNase inhibitor, and RNase-free water up to 20 $\mu \mathrm{L}$ were mixed. The mixture was incubated on ice for $10 \mathrm{~min}$ and at $42^{\circ} \mathrm{C}$ for $1 \mathrm{~h}$. Finally, the products were inactivated at $75^{\circ} \mathrm{C}$ for $5 \mathrm{~min}$ and then transferred to ice. Based on the previously cloned cDNA sequence, including KiSS-1 (JQ2319667), GPR54 (KF006323), GnRH (KC608826), and the $\beta$-actin gene (as an internal reference), the specific primer sequences were designed and synthesized by Biotechnology company (Sunbiotech Co., Ltd., Beijing, China). The primer sequences are shown in Table 1. 
Table 1. Primer sequences for real-time polymerase chain reaction in this study.

\begin{tabular}{llc}
\hline Name of primers & Primer sequences (5'-3') & Length of products (bp) \\
\hline KiSS-1 & F: ACTCATCAATGCCTGGGAAAAG & 222 \\
GPR54 & R: CCGAAGGAGTTCAGTTGTAGG & 213 \\
& F: CAACTTCTACATCGCCAACCT & \\
RnRH & R:AGGGGACACAGTCACATAC & 109 \\
Actin & R: GAGTCCTATGGGTTGCG & \\
& F: CAGGGCTGGGTTCTGCT & 221 \\
\hline
\end{tabular}

Real-time quantitative analysis was carried out using the Mx3000P real-time PCR instrument with synthesized cDNA as the template and SYBR Green QPCR Master Mix (Qiagen, Rui's Trading Co. Ltd., Jinan, China) as the fluorescent dye. The $20-\mu \mathrm{L}$ reaction system included $9 \mu \mathrm{L}$ SYBR Green Mix, $0.3 \mu \mathrm{L}$ ROX, $0.4 \mu \mathrm{L} 10 \mu \mathrm{M}$ each primer, and $\mathrm{ddH}_{2} \mathrm{O}$ up to $20 \mu \mathrm{L}$. Reaction conditions were as follows: pre-denaturation at $95^{\circ} \mathrm{C}$ for $10 \mathrm{~min}$, followed 40 cycles of denaturation at $95^{\circ} \mathrm{C}$ for $30 \mathrm{~s}$, annealing at $59^{\circ} \mathrm{C}$ for $45 \mathrm{~s}$, and elongation at $72^{\circ} \mathrm{C}$ for $45 \mathrm{~s}$, followed by 1 cycle of denaturation at $95^{\circ} \mathrm{C}$ for $1 \mathrm{~min}$, annealing at $59^{\circ} \mathrm{C}$ for $30 \mathrm{~s}$, and elongation at $95^{\circ} \mathrm{C}$ for $30 \mathrm{~s}$. At the end of each cycle, the amount of amplification product was calculated by measuring the fluorescence.

\section{Statistical analysis}

Differences in the serum melatonin concentration of striped hamsters treated with different photoperiods were analyzed using SPSS software (SPSS, Inc., Chicago, IL, USA). For striped hamsters treated with different photoperiods and melatonin injection, differences in the relative expression quantity of KiSS-1/GPR54 and GnRH were analyzed, and the correlations between the relative expression quantity of KiSS-1/GPR54 and $\mathrm{GnRH}$ in the hypothalamus were analyzed using SPSS software.

\section{RESULTS}

\section{Determination of melatonin concentrations}

Melatonin concentrations in the blood of the striped hamsters treated with different photoperiods are shown in Figure 1. Significant differences $(P<0.01)$ were observed in the melatonin concentrations of the blood between the striped hamsters treated with different photoperiods (Figure 1A). LD individuals showed the lowest melatonin content, while the SD group showed the highest; melatonin content in the CD group was between that of the LD and SD groups (Figure 1A). Significant differences $(P<0.05)$ were also observed between the female and male individuals in LD, but not in $C D$ and $S D$ individuals (Figure 1B). These results suggest that in striped hamsters, photoperiod regulates the synthesis and secretion of melatonin.

\section{Differential expression of KiSS-1 and GPR54 in the striped hamsters treated with different photoperiods}

Significant differences $(P<0.05)$ in the relative expression level of KiSS-1 in the hypothalamus, ovaries, and testicles were observed among striped hamsters treated with different 
photoperiods (Figure 2A and B); however, no significant difference $(P>0.05)$ in the hypothalamus was observed between the $C D$ and $S D$ female individuals and between the $C D$ and $L D$ male groups (Figure $2 A$ and $B$ ). Significant differences $(P<0.05)$ were observed in the relative expression levels of GPR54 in the hypothalamus and ovaries of female individuals when the CD group was compared with the LD and SD groups (Figure $2 \mathrm{C}$ ). Female individuals in the $C D$ group showed significantly higher relative expression levels of GPR54 in the hypothalamus $(P<0.05)$ and ovaries $(P<0.01)$ than female individuals in the SD and LD groups (Figure $2 C$ ), indicating that relative expression levels of GPR54 in the hypothalamus and ovary differed with the photoperiod. This suggests that the photoperiod is not the only factor regulating GPR54 expression in females. The relative expression level of GPR54 in the hypothalamus of CD male individuals was significantly lower $(P<0.01)$ than in $S D$ and $L D$ male individuals (Figure $2 D)$, and a significant difference $(P<$ 0.05 ) in the relative expression level of GPR54 in the hypothalamus was observed between the SD and LD male individuals (Figure 2D). In the testes, the relative expression level of GPR54 in male SD individuals was significantly lower $(P<0.05)$ than in male CD and LD individuals, and no significant differences $(P>0.05)$ in the relative expression quantity of GPR54 in the testis were observed between male CD and LD individuals (Figure 2D).

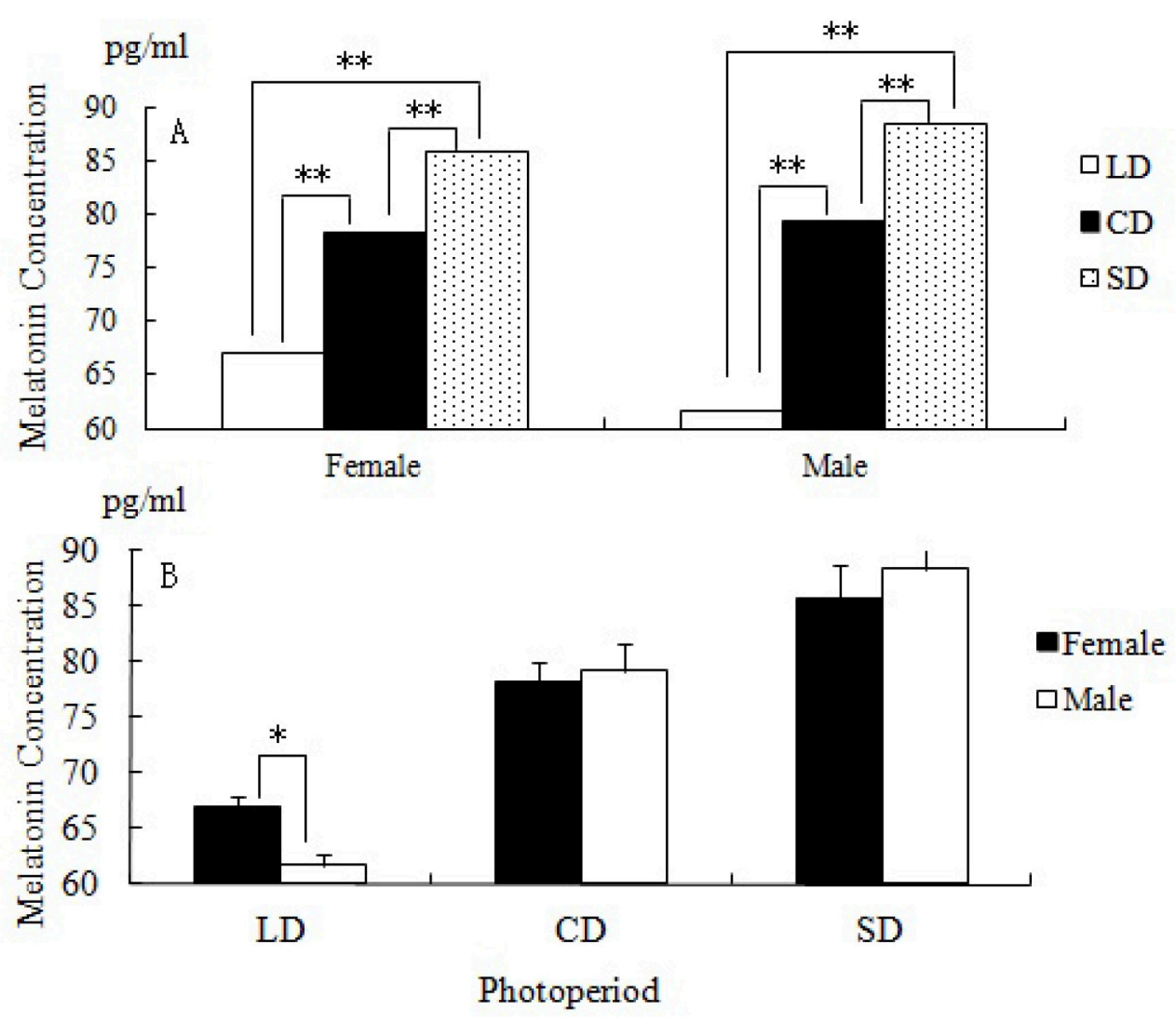

Figure 1. Serum melatonin concentrations in striped hamsters treated with different photoperiods. $L D, C D$, and SD refer to photoperiods of $16 \mathrm{~L}: 8 \mathrm{D}, 12 \mathrm{~L}: 12 \mathrm{D}$, and $8 \mathrm{~L}: 16 \mathrm{D}$, respectively. $\left({ }^{*} \mathrm{P}<0.05\right.$; $\left.{ }^{*} \mathrm{P}<0.01\right)$. 

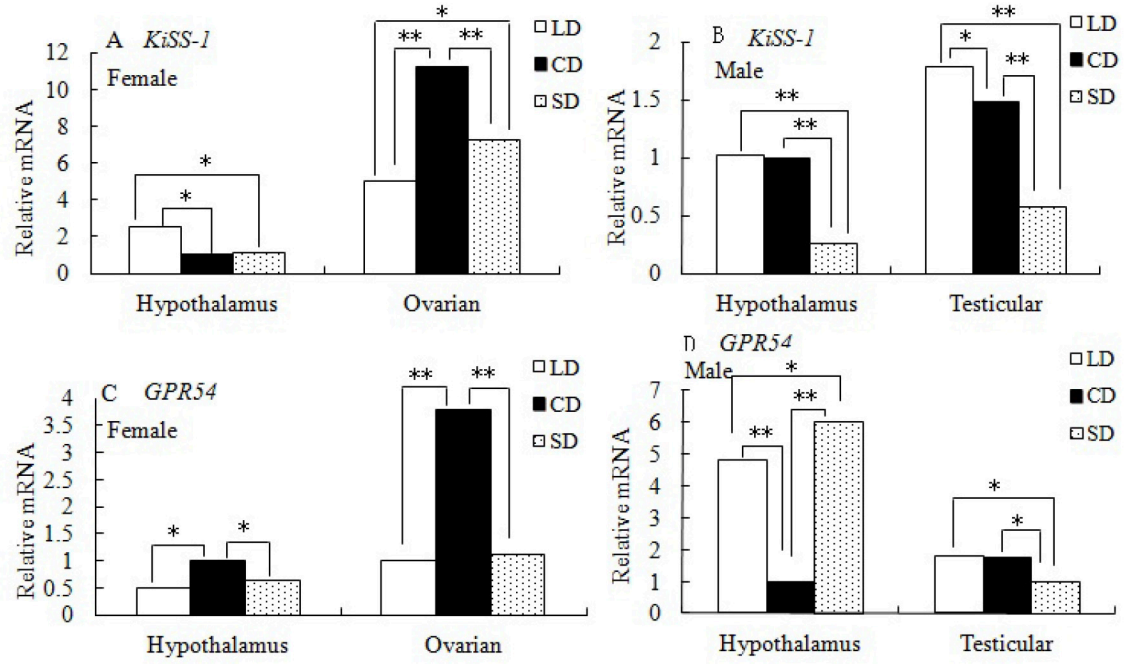

Figure 2. Comparison of the relative expression of KiSS-1 and GPR54 in striped hamsters treated with different photoperiods. The expression quantity of KiSS-1 and GPR54 in the hypothalamus of CD individuals is assumed to be 1.

These results demonstrated that the relative expression level of KiSS-1 in the hypothalamus of male and female individuals and the testicles of the male individuals was consistent with the photoperiods, while that in the ovarian of female individuals showed differences with the photoperiods, indicating that further complicated regulatory mechanisms of KiSS-1 expression may exist in the ovary. The relative expression level of GPR54 in the hypothalamus and gonads of the male and female striped hamsters was inconsistent with the photoperiods, suggesting that the photoperiod regulates the expression of GPR54 in a more complicated mechanism or is not directly involved in the regulation of GPR54 expression.
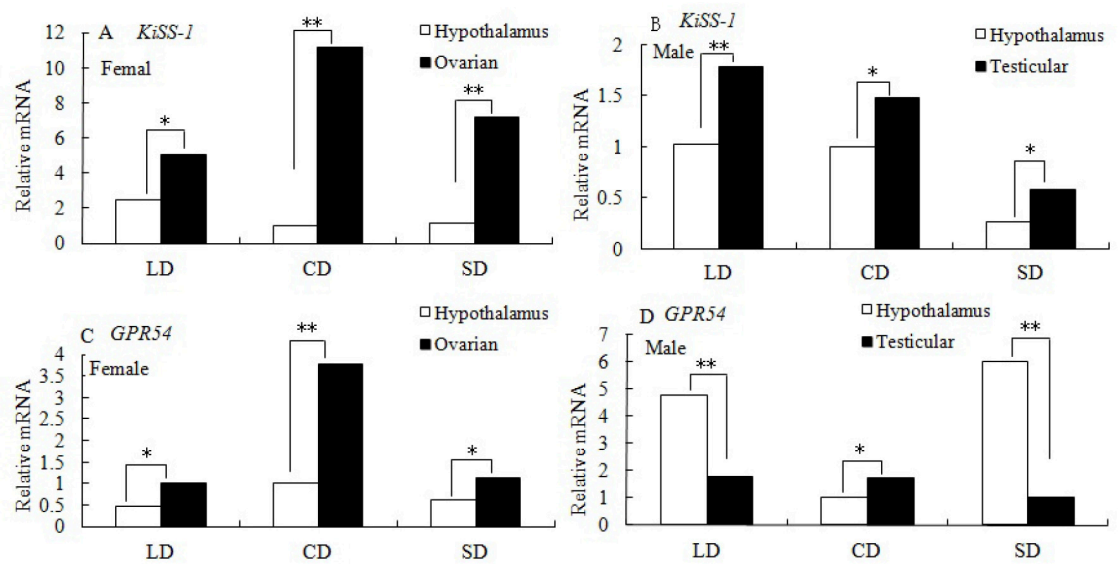

口Hypothalamus

Figure 3. Comparison of relative expression levels of KiSS-1 and GPR54 in the hypothalamus with those in the gonads of male and female striped hamsters treated for the same photoperiod. The expression quantity of KiSS-1 and GPR54 in the hypothalamus of CD individuals is assumed to be 1 . 
The relative expression level of KiSS-1 in the hypothalamus was significantly lower ( $P$ $<0.01$ ) than in the ovaries or testicles of female or male striped hamsters treated with the same photoperiod (Figure $3 \mathrm{~A}$ and $\mathrm{B}$ ). For the female striped hamsters treated with the same photoperiod, significantly higher expression of GPR54 $(P<0.05)$ was observed in the ovaries than in the hypothalamus (Figure $3 C$ ), and the same results were observed for male hamsters treated with the CD period, but not for male hamsters treated with LD and SD photoperiods (Figure 3D). GPR54 expression in the testicles was significantly lower $(P<0.01)$ than that in the hypothalamus in male hamsters treated with the LD and SD photoperiod (Figure 3D). These results suggest that the role of kisspeptin encoded by KiSS-1 was more significant in ovaries or testicles than in the hypothalamus in male and female striped hamsters, but GPR54 has a more significant role in gonads than in the hypothalamus only in females.

\section{Expression levels of KiSS-1/GPR54 and GnRH in striped hamsters injected with melatonin}

The relative expression levels of KiSS-1 in the hypothalamus and gonads of both female and male striped hamsters were significantly reduced $(P<0.01)$ when injected with melatonin (Figure 4A). The expression of GPR54 in the ovaries and testicles of striped hamsters were also significantly decreased $(P<0.01)$ after melatonin injection, with no change in expression levels of GPR54 in the male hypothalamus after melatonin injection (Figure 4B). In the hypothalamus of female striped hamsters, GPR54 expression was significantly increased $(P<0.05)$ following melatonin injection (Figure 4B). These results further illustrate that melatonin decreased KiSS1 expression in the hypothalamus and gonads of striped hamsters, and reduced GPR54 expression in the ovaries and testicles of striped hamsters and increased GPR54 expression in the hypothalamus. In addition, relative $\mathrm{GnRH}$ expression in the hypothalamus of striped hamsters was significantly decreased $(P<0.05)$ after melatonin injection.
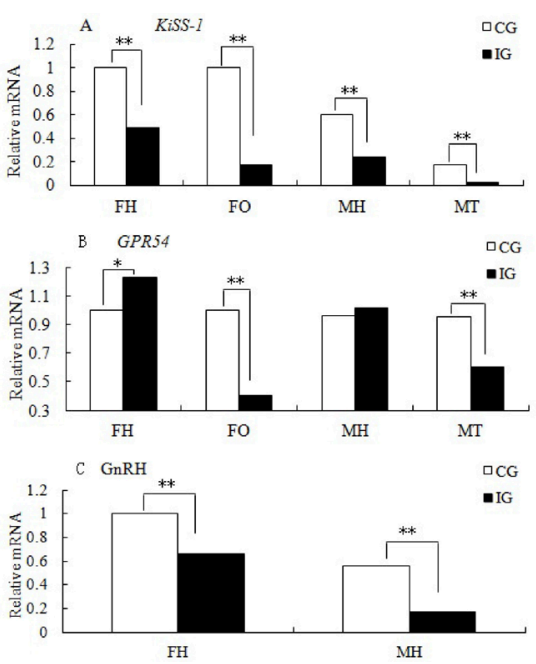

Figure 4. Comparison of expression levels of KiSS-1/GPR54 and GnRH in striped hamsters injected with melatonin. $\mathrm{FH}$, hypothalamus of the female striped hamsters; FO, ovaries of female striped hamsters; $\mathrm{MH}$, hypothalamus of male striped hamsters; MT, testicles of male striped hamsters; IG, injection group; CG, control group. The expression quantity of KiSS-1 and GPR54 in the hypothalamus or ovaries of CG female individuals is assumed to be 1. 


\section{Correlation between relative KiSS-1 and GPR54 levels with GnRH expression}

The correlation between relative KiSS-1 and GPR54 expression levels and GnRH expression in the hypothalamus was analyzed using SPSS. The results showed that KiSS-1 expression in the hypothalamus was positively correlated with that of $G n R H$ in the hypothalamus ( $r=0.811, P=0.039$ ), and a negative correlation was observed between relative GPR54 and GnRH expression levels in the hypothalamus $(r=-0.733, P=0.017)$ in female striped hamsters. A significantly positive correlation $(r=0.863, P=0.047)$ in relative expression between KiSS-1 and $\mathrm{GnRH}$ in the hypothalamus was observed, while no significant correlation $(r=-0.291, \mathrm{P}=0.359)$ was observed between the relative expression of GPR54 in the hypothalamus and that of $\mathrm{GnRH}$ in the hypothalamus in male striped hamsters. These results suggest that KiSS-1 expression increases expression of $\mathrm{GnRH}$, which is a main factor regulating animal reproduction. GPR54 may have a negative effect on $G n R H$ expression in the hypothalamus.

\section{DISCUSSION}

\section{Correlations between photoperiod and melatonin}

Melatonin is released during the dark phase to ensure that high levels of this hormone accumulate in the plasma and cerebrospinal fluid at night and remain low during the day. Melatonin mediates the central effects of the photoperiod in the neuroendocrine circuitry controlling pituitary functions. Melatonin is also involved in the seasonal control of reproduction (Reiter, 1993). More specifically, melatonin is a factor that mediates the actions of photoperiod signals in the regulation of gonadal development, such that seasonal changes in day-length are transduced into the appropriate melatonin rhythms, which in turn regulate the brain-pituitary-gonad axis. Melatonin concentrations in the blood were in consistent with the photoperiod experienced by striped hamsters.

\section{Photoperiod regulates KiSS-1 and GPR54 expression}

Kisspeptin was proposed to be a main mediator for photoperiod in the reproductive system. The acting process of photoperiod is mainly mediated by melatonin, which is secreted by the pineal gland at nighttime. Differential expression of KiSS-1 regulated by photoperiods in the hypothalamus of the hamsters has been extensively studied. For example, KiSS-1 expression in the hypothalamus of Syrian hamsters was higher under LD conditions than under SD conditions. When Syrian hamsters were maintained with SD for 8-10 weeks, the level of KiSS-1 mRNA (Revel et al., 2006b) and the number of KiSS-1-expressing neurons were markedly reduced in the Arc (Simonneaux et al., 2009). However, different results were observed in Siberian hamsters than in Syrian hamsters.

In the SD Siberian hamster, KiSS-1 expression decreased in the Arc and increased in the AVPV, indicating that the photoperiodic effect on KiSS-1 expression is spatial-conditional. Additionally, the hypothalamus of the ovariectomized ewe showed higher expression of KiSS1 in the Arc during the SD period than during LD (Smith et al., 2007). This suggests that the expression of KiSS-1 in the hypothalamus of the ewe is regulated by the sex hormones except for the photoperiod. These studies suggest that the photoperiodic effect on KiSS-1 expression is species-dependent. 


\section{Differential expression of KISS-1 and GPR54 among tissues and sexes}

KiSS-1 is expressed in a wide range of tissues in addition to the hypothalamus, ovaries, and testicles (Kitahashi and Parhar, 2013). KiSS-1 expression in the Arc was downregulated in SD Syrian hamsters and upregulated in SD Siberian hamsters. Acute kisspeptin injection in Siberian hamster triggered LH release under both LD and SD conditions in males (Greives et al., 2008), but only under SD conditions in females (Mason et al., 2007).

\section{Melatonin regulates KiSS-1 and GPR54 expression}

The expression level of KiSS-1 in the Arc of Syrian hamsters increased when the pineal glands were resected (Ansel et al., 2010), indicating that melatonin could restrict the expression of KiSS-1 in the Arc of the Syrian hamsters. Melatonin reduced KiSS-1 expression in the hypothalamus, ovaries, and testicles in striped hamsters, reduced GPR54 expression in the ovaries and testicles of striped hamsters, and increased GPR54 expression in the hypothalamus. KiSS-1 expression was positively correlated with $G n R H$ expression in the hypothalamus of striped hamsters, while GPR54 expression was negatively correlated.

SD conditions increased the secretion of melatonin, which inhibits the expression of KiSS1 , and thus reduce the secretion of $\mathrm{GnRH}$ to drive the reproductive axis. In contrast, LD increased the expression of KiSS-1 via reduce the secretion of melatonin; KiSS-1 regulates GnRH during reproductive activity. The effect of melatonin on the expression of GPR54 differed among the tissues and sexes. Photoperiods regulate kisspeptin genes via a melatonin-dependent integrative mechanism. Our results suggested that stronger production of melatonin in SD inhibits KiSS-1 expression and therefore reproductive activity. The finding that photoperiodic variation in melatonin regulates KiSS-1 expression in the Arc of male Syrian hamsters suggested that kisspeptin relays the melatonin signal to the reproductive axis to reduce production of kisspeptin in SD hamsters. This may be responsible in part for the inhibition of reproductive activity. The photoperiod may modulate KiSS-1 neurons to drive the reproductive axis via melatonin.

\section{Conflicts of interest}

The authors declare that there are no conflicts of interest.

\section{ACKNOWLEDGMENTS}

Research financially supported by the National Natural Science Foundation of China (Grant \#31070332, \#31270417, and \#31300304).

\section{REFERENCES}

Ansel L, Bolborea M, Bentsen AH, Klosen P, et al. (2010). Differential regulation of kiss1 expression by melatonin and gonadal hormones in male and female Syrian hamsters. J. Biol. Rhythms 25: 81-91.

Bartke A and Steger RW (1992). Seasonal changes in the function of the hypothalamic-pituitary-testicular axis in the Syrian hamster. Proc. Soc. Exp. Biol. Med. 199: 139-148.

Clarkson J and Herbison AE (2006). Postnatal development of kisspeptin neurons in mouse hypothalamus; sexual dimorphism and projections to gonadotropin-releasing hormone neurons. Endocrinology 147: 5817-5825. 
de Roux N, Genin E, Carel JC, Matsuda F, et al. (2003). Hypogonadotropic hypogonadism due to loss of function of the KiSS1derived peptide receptor GPR54. Proc. Natl. Acad. Sci. U. S. A. 100: 10972-10976.

Dhillo WS, Chaudhri OB, Patterson M, Thompson EL, et al. (2005). Kisspeptin-54 stimulates the hypothalamic-pituitary gonadal axis in human males. J. Clin. Endocrinol. Metab. 90: 6609-6615.

Franceschini I, Lomet D, Cateau M, Delsol G, et al. (2006). Kisspeptin immunoreactive cells of the ovine preoptic area and arcuate nucleus co-express estrogen receptor alpha. Neurosci. Lett. 401: 225-230.

Funes S, Hedrick JA, Vassileva G, Markowitz L, et al. (2003). The KiSS-1 receptor GPR54 is essential for the development of the murine reproductive system. Biochem. Biophys. Res. Commun. 312: 1357-1363.

Goldman BD (2001). Mammalian photoperiodic system: formal properties and neuroendocrine mechanisms of photoperiodic time measurement. J. Biol. Rhythms 16: 283-301.

Gottsch ML, Cunningham MJ, Smith JT, Popa SM, et al. (2004). A role for kisspeptins in the regulation of gonadotropin secretion in the mouse. Endocrinology 145: 4073-4077.

Greives TJ, Kriegsfeld LJ and Demas GE (2008). Exogenous kisspeptin does not alter photoperiod-induced gonadal regression in Siberian hamsters (Phodopus sungorus). Gen. Comp. Endocrinol. 156: 552-558.

Greives TJ, Mason AO, Scotti MA, Levine J, et al. (2007). Environmental control of kisspeptin: implications for seasonal reproduction. Endocrinology 148: 1158-1166.

Irwig MS, Fraley GS, Smith JT, Acohido BV, et al. (2004). Kisspeptin activation of gonadotropin releasing hormone neurons and regulation of KiSS-1 mRNA in the male rat. Neuroendocrinology 80: 264-272.

Kanda S, Akazome Y, Matsunaga T, Yamamoto N, et al. (2008). Identification of KiSS-1 product kisspeptin and steroidsensitive sexually dimorphic kisspeptin neurons in medaka (Oryzias latipes). Endocrinology 149: 2467-2476.

Kauffman AS, Clifton DK and Steiner RA (2007). Emerging ideas about kisspeptin- GPR54 signaling in the neuroendocrine regulation of reproduction. Trends Neurosci. 30: 504-511.

Kitahashi T, Ogawa S and Parhar IS (2009). Cloning and expression of kiss2 in the zebrafish and medaka. Endocrinology 150: 821-831.

Kitahashi T and Parhar IS (2013). Comparative aspects of kisspeptin gene regulation. Gen. Comp. Endocrinol. 181: 197-202.

Li S, Liu B and Xu D (1990). Studies on the relationship between chromosome No15 and the biological characteristics of malignant transformed Syrian hamster fibroblast cell lines. Proc. Chin. Acad. Med. Sci. Peking Union Med. Coll. 5: 43-46.

Malpaux B, Tricoire H, Mailliet F, Daveau A, et al. (2002). Melatonin and seasonal reproduction: understanding the neuroendocrine mechanisms using the sheep as a model. Reprod. Suppl. 59: 167-179.

Mason AO, Greives TJ, Scotti MA, Levine J, et al. (2007). Suppression of kisspeptin expression and gonadotropic axis sensitivity following exposure to inhibitory day lengths in female Siberian hamsters. Horm. Behav. 52: 492-498.

Matsui H, Takatsu Y, Kumano S, Matsumoto H, et al. (2004). Peripheral administration of metastin induces marked gonadotropin release and ovulation in the rat. Biochem. Biophys. Res. Commun. 320: 383-388.

Messager S, Chatzidaki EE, Ma D, Hendrick AG, et al. (2005). Kisspeptin directly stimulates gonadotropin-releasing hormone release via G protein-coupled receptor 54. Proc. Natl. Acad. Sci. U. S. A. 102: 1761-1766.

Navarro VM, Castellano JM, Fernández-Fernández R, Tovar S, et al. (2005). Effects of KiSS-1 peptide, the natural ligand of GPR54, on follicle-stimulating hormone secretion in the rat. Endocrinology 146: 1689-1697.

Parhar I, Ogawa S and Kitahashi T (2012). RFamide peptides as mediators in environmental control of GnRH neurons. Prog. Neurobiol. 98: 176-196.

Parhar IS, Ogawa S and Sakuma Y (2004). Laser-captured single digoxigenin-labeled neurons of gonadotropin-releasing hormone types reveal a novel G protein-coupled receptor (Gpr54) during maturation in cichlid fish. Endocrinology 145: 3613-3618.

Pévet $\mathrm{P}$ (1988). The role of the pineal gland in the photoperiodic control of reproduction in different hamster species. Reprod. Nutr. Dev. 28: 443-458.

Prendergast BJ (2005). Internalization of seasonal time. Horm. Behav. 48: 503-511.

Reiter RJ (1993). The melatonin rhythm: both a clock and a calendar. Experientia 49: 654-664.

Revel FG, Saboureau M, Masson-Pévet M, Pévet P, et al. (2006a). KiSS-1: a likely candidate for the photoperiodic control of reproduction in seasonal breeders. Chronobiol. Int. 23: 277-287.

Revel FG, Saboureau M, Masson-Pévet M, Pévet P, et al. (2006b). Kisspeptin mediates the photoperiodic control of reproduction in hamsters. Curr. Biol. 16: 1730-1735.

Roa J and Tena-Sempere M (2007). KiSS-1 system and reproduction: comparative aspects and roles in the control of female gonadotropic axis in mammals. Gen. Comp. Endocrinol. 153: 132-140.

Servili A, Le Page Y, Leprince J, Caraty A, et al. (2011). Organization of two independent kisspeptin systems derived from evolutionary-ancient kiss genes in the brain of zebrafish. Endocrinology 152: 1527-1540.

Shahab M, Mastronardi C, Seminara SB, Crowley WF, et al. (2005). Increased hypothalamic GPR54 signaling: a potential mechanism for initiation of puberty in primates. Proc. Natl. Acad. Sci. U. S. A. 102: 2129-2134. 
Simonneaux V, Ansel L, Revel FG, Klosen P, et al. (2009). Kisspeptin and the seasonal control of reproduction in hamsters. Peptides 30: 146-153.

Simonneaux V and Ribelayga C (2003). Generation of the melatonin endocrine message in mammals: a review of the complex regulation of melatonin synthesis by norepinephrine, peptides, and other pineal transmitters. Pharmacol. Rev. 55: 325-395.

Smith JT, Clay CM, Caraty A and Clarke IJ (2007). KiSS-1 messenger ribonucleic acid expression in the hypothalamus of the ewe is regulated by sex steroids and season. Endocrinology 148: 1150-1157. 